\title{
GNARE: Automated System for High-Throughput Genome Analysis with Grid Computational Backend*
}

\author{
Dinanath Sulakhe ${ }^{1}$ Alex Rodriguez ${ }^{1}$ Mark D’Souza ${ }^{1}$ Michael Wilde ${ }^{1}$ Veronika Nefedova $^{1}$ Ian Foster $^{1,2}$ Natalia Maltsev $^{1}$ \\ ${ }^{1}$ Mathematics and Computer Science Division, Argonne National Laboratory, Argonne, IL 60439, USA \\ ${ }^{2}$ Department of Computer Science, University of Chicago, Chicago, IL 60637, USA
}

\begin{abstract}
Recent progress in genomics and experimental biology has brought exponential growth of the biological information available for computational analysis in public genomics databases. However, applying the potentially enormous scientific value of this information to the understanding of biological systems requires computing and data storage technology of an unprecedented scale. The Grid, with its aggregated and distributed computational and storage infrastructure, offers an ideal platform for high-throughput bioinformatics analysis. To leverage this we have developed the Genome Analysis Research Environment (GNARE) - a scalable computational system for the highthroughput analysis of genomes, which provides an integrated database and computational backend for datadriven bioinformatics applications. GNARE efficiently automates the major steps of genome analysis including acquisition of data from multiple genomic databases; data analysis by a diverse set of bioinformatics tools; and storage of results and annotations.

High-throughput computations in GNARE are performed using distributed heterogeneous Grid computing resources such as Grid2003, TeraGrid, and the DOE Science Grid. Multi-step genome analysis workflows involving massive data processing, the use of application-specific tools and algorithms and updating of an integrated database to provide interactive web access to results are all expressed and controlled by a "virtual data" model which transparently maps computational workflows to distributed Grid resources. This paper describes how Grid technologies such as Globus, Condor, and the Gryphyn Virtual Data System were applied in the development of GNARE. It focuses on our approach to Grid resource allocation and to the use of GNARE as a computational framework for the development of bioinformatics applications.
\end{abstract}

\section{Introduction}

During the past decade, the scientific community has witnessed an unprecedented accumulation of gene sequence data and data related to the physiology and biochemistry of organisms. To date, 250 genomes have been sequenced and genomes of more than 1000 organisms are at various levels of completion [1]. In order to exploit the enormous scientific value of this information for understanding biological systems, the information must be integrated, analyzed, graphically displayed and ultimately modeled computationally [2].

Comparative and evolutionary analysis of wide spectrum of phylogenetically diverse organisms represents one of the most powerful approaches for interpretation of genomes and understanding mechanisms of adaptation of organisms to environments. It allows for systematic exploration of adaptive mechanisms that led to diversification of biological systems, on all levels of their organization: genomic, metabolic, and phenotypic. However such approach requires the development of high-throughput computational environments that integrate (i) large amounts of genomic and experimental data (ii) powerful tools and algorithms for knowledge discovery and data mining (iii) tools for collaborative analysis of biological data by the experts residing in remote locations and (iv) scalable computational backend.

The efficiency and accuracy of genetic sequence analysis is achieved by the use of a variety of bioinformatics tools and algorithms (e.g., analysis of global similarities [3] [4] [5], domain and motif analysis [6] [7] [8], analysis of the relevant structural [9] [10] and functional data). The process of acquisition and integration of needed information can be extremely tedious, time-consuming, and prone to human error if done by manually scheduled computations. Reliable execution of such multi-step analytical processes could be achieved by implementation of controlled workflows and analytical pipelines. Another problem that emerges in high-throughput bioinformatics is related to the fact that most of the tools and algorithms used for analysis of genomic data are CPU-intensive, requiring computational resources beyond those available to researchers at a single location. The aggregated and distributed computational and storage infrastructure of the Grid offers an ideal platform for mining biological information at this large scale. There are a number of groups working on utilizing

\footnotetext{
* Based on "GNARE: An Environment for Grid-Based High-Throughput Genome Analysis” by D. Sulakhe, A. Rodriguez, M. D’Souza, M. Wilde, V. Nefedova, I. Foster, and N. Maltsev which appeared in Proceedings of CCGRID 2005. (C2005 IEEE.
} 
grid technologies for bioinformatics purposes, including the Integrative Genome Annotation Pipeline (iGAP) which has been used by the international consortium "Encyclopedia of Life" [11] for the extensive annotation of protein sequence data, myGrid [12] - a large scale Grid-based European effort, North Carolina BioGRID [13], EUROGRID [14], and the Asia Pacific BioGrid Initiative [15].

We have developed, and continue to extend, a system that utilizes Grid technology for the needs of highthroughput genetic sequence analysis. This Genome Analysis Research Environment (GNARE) is a highperformance scalable computational environment that allows efficient automation of the major steps of genome analysis, including data acquisition from a variety of genomic databases and its analysis by several bioinformatics tools and algorithms. GNARE also expedites the process of storing the results of analyses and annotations. It is based on Grid technology (specifically Globus [16], Condor [17], and the GriPhyN virtual data system [18]) and uses the computational resources of GRID2003 [19], TeraGrid [20], and the DOE science Grid [21] to perform high-throughput computations. GNARE's flexible architecture allows users to tailor the genome analysis process to their individual needs and requirements. It can function in an automated mode as well as interactively through a webbased interface.

This paper describes the GNARE implementation and its automated database update pipeline GADU, as well as our experiences using it on the Grid, and increasing the application's computational power and speed through further Grid integration and enhancement. We also describe scientific applications that use GNARE for highthroughput analysis and annotation of genomes.

\section{System Overview and Design}

GNARE comprises three main components, as illustrated in Figure 1: GNARE Architecture. GADU is the main engine that executes computationally intensive workflows on the Grid and performs the Integrated Database updates. The Integrated Database (see section 4) warehouses sequence data and annotations from the monitored public databases as well as the results of data analyses from the GADU update engine. The third component is the set of web-based applications that use the Integrated Database (see section 5.2) and GADU's analysis services.

GADU serves as the heart of the system. It acts as a gateway to the Grid, handling all computational analysis for the GNARE system. It is an automated, scalable, highthroughput computational workflow engine that enables the Grid execution of bioinformatics tools. The interpretation of every newly-sequenced genome involves the analysis of sequence data by a workflow pipeline composed of multiple bioinformatics tools, the execution of result and annotation parsers, and other intermediate data-transforming scripts. The GADU implementation comprises two modules, and Analysis Server and an Update Server. The Analysis Server automatically creates workflows in the abstract Virtual Data Language, based on predefined templates (Section 3.5), which it then executes on distributed Grid resources such as Grid2003 and TeraGrid. The Update Server updates the Integrated Database with recently changed data from a set of monitored public databases (currently including NCBI RefSeq [22], PIR [23], InterPro [6], and KEGG [24]).

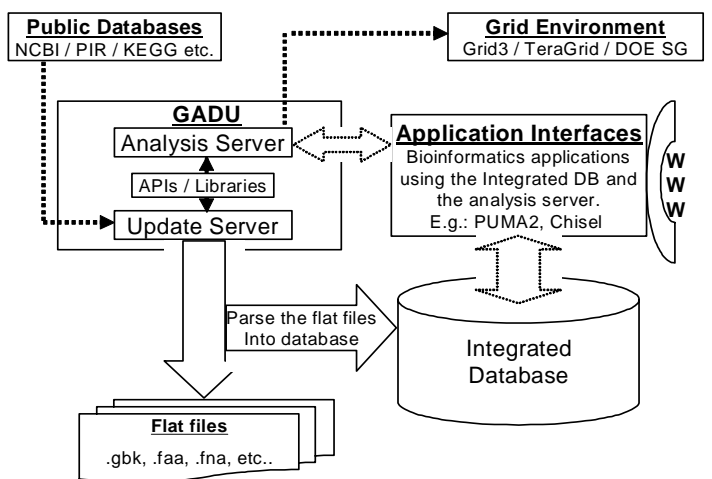

Figure 1: GNARE Architecture

In the following sections, we describe the implementation details of each of the components of GNARE.

\section{The GADU Analysis Server}

We start with the GADU Analysis Server, which is responsible for executing bioinformatics analyses on the Grid. Its components are shown in Figure 2 and described below.

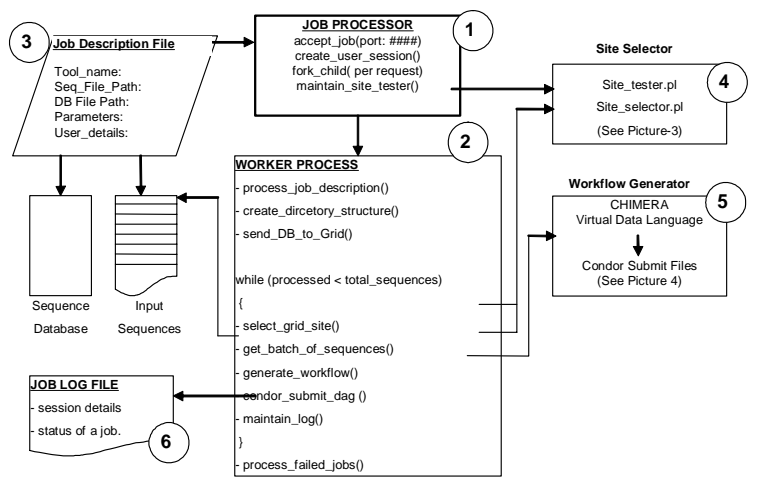

Figure 2: GADU Analysis Server 


\subsection{Job Description File}

The job description file (item 3 in Figure 2) describes a job that may involve simply running a bioinformatics tool on the local machine, or alternatively the execution of a predefined complex workflow on the Grid.

For example, the analysis of a set of protein sequences using BLAST [25] against some database is represented in the Job Description File with all the information required to perform this analysis, like the tool name (BLAST), path of the input sequence file, path of the database sequence file, parameters to be used for BLAST. Similarly, every analysis (e.g., BLOCKS [26], PFAM [27], TMHMM [28] etc.) can be represented by a Job Description File appropriate for the tool. Using the information from this file, the Job Processing Server along with Workflow Generator creates the actual workflow in a Virtual Data Language and eventually in the form of a condor DAG.

\subsection{Job Processing Server}

The Job Processing Server (item 1 in Figure 2) accepts a Job Description File and creates a worker process (item 2) to handle the job. Other stand-alone services are also invoked at this time, namely the Site Selector (item 4) and Workflow Generator (item 5). In addition to creating the worker process, the server also takes care of creating a session for each job and also controls the "Site Selector" so as to keep an updated list of good working sites for job submission. The site selector is explained later.

\subsection{Worker Process}

The worker process (item 2 in Figure 2) determines how to handle each job based on the information in the Job Description File. The worker process first creates the directory structure for the job and then sends the sequence database (e.g., in the case of BLAST) to all usable sites on the Grid. A list of usable sites is collected from the "Site Selector" (item 4).

The next step is to create a batch job where we select a batch of query sequences to be submitted to a selected Grid site. The worker process first asks for a "good performing site" from the site selector. Based on the information it gets back (explained in next subsection) it picks a batch of sequences from the original input sequence file and sends them to the site selected. For example, if the Site Selector picked a site with 20 CPUs to be used for 1000 sequences to be processed for BLAST, then the worker process would pick a batch of 1000 sequences from the main input file after the last sequence processed. Then the worker process calls the Workflow Generator (item 5 in Figure 2, described in detail in Section 3.5) which encodes the workflow in an abstract Virtual Data Language (VDL). Condor represents a workflow in the form of a DAG and executes the workflow on the selected Grid site. Once the Worker Process submits a batch job or rather a Condor DAG to the selected grid site using Condor, it writes all the details of the job to a log file (item 6 in Figure 2), and goes back to generate the next batch job. The worker process continues to do this till all sequences have been processed.

\subsection{Site Selector}

One challenge in using the Grid reliably for highthroughput analysis is monitoring the state of all Grid sites and how well they have performed for job requests from a given submit host. If we are executing a workflow that may submit large numbers of jobs to different Grid sites over a period of several days, it is important to keep track of which sites are available to run jobs at different times. We view a site as "available" if (i) our submit host can communicate with it, (ii) it is responding to Globus job-submission commands, and (iii) it will run our jobs promptly, with minimal queuing delays.

To address this issue, we have developed a Site Selector (item 4 in Figure 2, and also Figure 3) that uses information collected at the submit host to determine which sites meet the required responsiveness criteria.

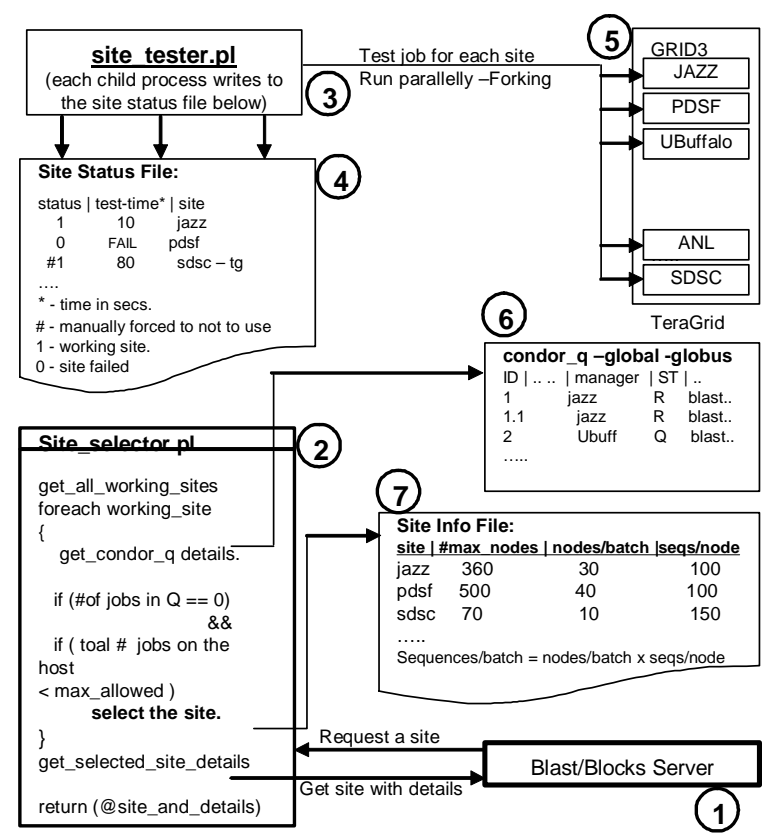

Figure 3: Implementation of the site selector

The site tester ("probe") script (item 3 in Figure 3) can be started and controlled manually or by the Job Processing Server. The script submits a small test job to all Grid sites of interest and then records for each site whether or not the site responded and, if so, its 
performance (i.e., response time). The resulting Site Status File (item 4) notes for each site a status (either the site responded correctly, or the site responded incorrectly or failed to respond before a timeout period) and the time taken for the site to respond. We update this Site Status File regularly to maintain the current status of all the sites.

The site selector proper then uses the information in the Site Status File to select the site to which we can submit our next job. The selector scripts takes into consideration the state of the Condor queue at the submit host (item 6 in Figure 3) in order to make a decision. Whenever there is request from the 'Job Processing Server' to select a good site, the site selector selects all the sites from the Site Status File that have been flagged as acceptable by the tester script. For each site it then looks at the submit host's Condor-G queue (using the condor_q command) for the number of jobs that have been already submitted to that site. If all the previously submitted jobs to this site are in 'Running' state or if there are no jobs submitted to this site, then it selects the site for the next job. However, if there is even one job at this site that is waiting to run, then the site in consideration is not selected and the selector scripts looks at the next site. Once a job has been submitted, the selector script makes sure that the same site is not selected again for a specified period, so that the newly submitted job has time to show up in the Condor queue.

Apart from selecting a site, the site selector also returns job-specific information to the requester. Based on the statistics of the previously executed jobs, it creates a configuration file, the Site Info File (item 7). This file records the maximum number of nodes (or CPUs) at each site, the number of nodes to be requested for each batch job submitted at this site, the number of sequences to be processed at each node, and other site-specific information. Based on this configuration file, the selector script also calculates the number of sequences that should be processed in a single job at the selected site and the number of nodes to be requested for that job. This information is all returned to the requester, which in our case is the Worker Process.

\subsection{Workflow Generator}

Having determined the site to which a job should be directed, the worker process assembles a complete description of the job that is to be executed and passes this information to the Workflow Generator (item 5 in Figure 2). The Workflow Generator is then responsible for producing a workflow suitable for execution in the Grid environment. This task is accomplished through the use of the GriPhyN virtual data system's "virtual data language" (VDL) [29]. VDL provides simplified, abstract access to large-scale grid computation and storage resources, and also provides the ability to:

- track accurately the provenance of results of the workflows, describing how they were obtained from transformations of input data;

- discover data through tools that search for specific transformations;

- produce new analysis work based on previously executed work, which allows for the comparison of transformation patterns executed at different times; and

- $\quad$ audit and disseminate results.

Figure 4 illustrates the six-stage workflow produced for a simple comparative analysis of 100 protein sequences, grouped into 5 sets, through the BLAST tool. The six stages include the data transfers to and from Grid storage servers, partitioning input data for the subsequent BLAST process, parsing of specific information that the user wants to capture from protein sequences, and concatenation of final results.

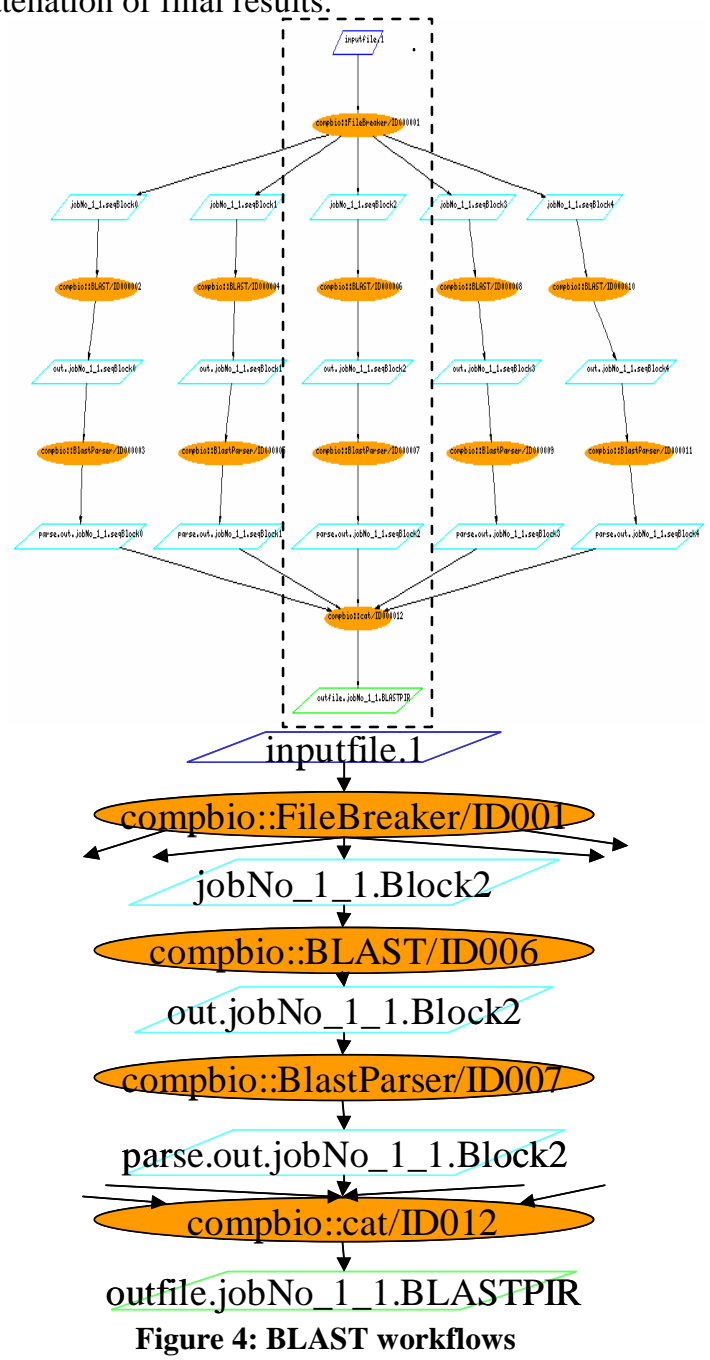

Top, a 6-step workflow with 5-way parallelism; below: the center-path details. 
This workflow is represented in VDL via a set of transformation definitions (abstract interfaces that describe an application program such as BLAST, Blocks, or result-parser) and a set of derivations-in effect, function calls that specify inputs such as genome sequence files, output files from comparative analysis tools, and textual parameters: i.e., all the information coming from the worker process.

The VDL fragments below illustrate the notation. The first two statements define the transformations FileBreaker and BLAST shown in Figure 4. These transformation definitions act as function definitions and specify the formal arguments to an application, and the details of how those arguments are passed to and from the application represented by the TR definition. In the case of FileBreaker, the arguments include a genome sequence input file and the subsequent output files, as well as the number of computer resources to use in the grid environment.

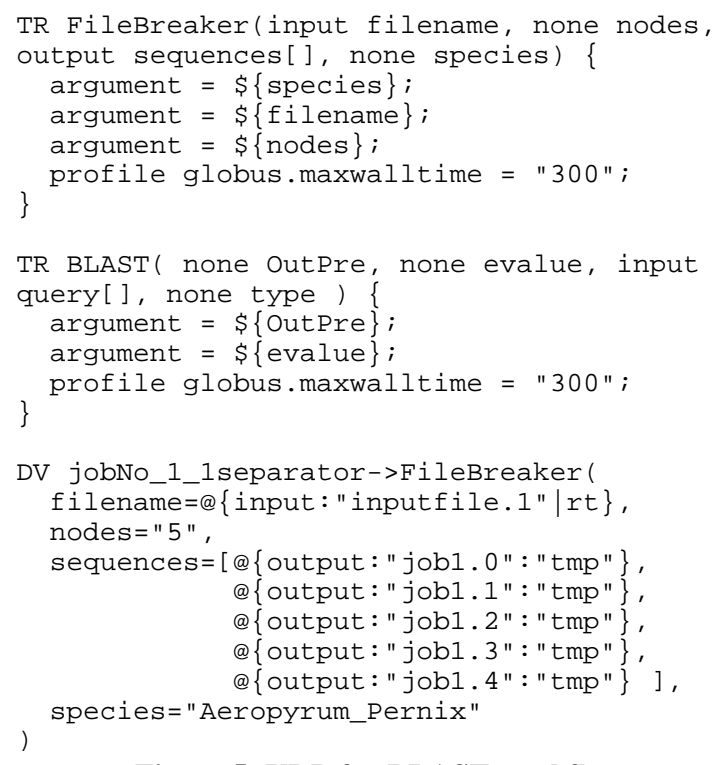

Figure 5: VDL for BLAST workflow

The third statement specifies a FileBreaker derivation. Derivations, defined by "DV" statements, specify the actual arguments to be passed to a transformation. File names used as arguments in DV statements are "logical names", mapped to physical file names at run time.

Data transfer for VDL is performed automatically and transparently for the user. For example, the physical file for the logical filename "inputfile.1" will be transferred automatically to the site selected for execution of the FileBreaker transformation via GridFTP [30], which provides secure, efficient data movement in Grid environments. Input files to transformations are automatically located in the Grid by searching for physical copies of a logical file in a replica location service such as RLS [31. Output files are automatically cataloged in the same location service for use in subsequent transformations and workflows. In the transformation "BLAST," above, we use the "profile" feature of VDL to specify the run-time limit for that process. VDL profiles permit parameters to be passed to components of the run-time environment.

Once the VDL for the workflow is written, the worker process invokes the GriPhyN virtual data system to execute the workflow at the Grid site selected previously by the site selector. This task is achieved via the DAGman (Directed Acyclic Graph Manager), a metascheduler for condor, which submits jobs to remote site via Condor-G in an order determined by specified interdependencies, which in this case are derived from the VDL specification.

\section{Update Server and Integrated Database}

As shown in Figure 1, the Update Server helps in the acquisition of a variety of genomic data from the public databases like NCBI, PIR, PDB [32], KEGG and parses and uploads the data into the integrated database. It also uploads the parsed results of the various analysis tools and workflows into the integrated database.

The Update Server uses its library of parsers for all the different databases (flat files) that it downloads from the public databases. The acquisition process can be automatically executed at a predefined interval of time, in which case the server checks for new updates to download, or it can be started manually whenever there is a new release of updated data. Once the databases are downloaded and parsed, the Update Server uploads the data into the integrated database. The uploading process has been parallelized locally by forking appropriate number (based on the maximum permitted number of database handles) of child processes that upload the data using SQLLoader. Similarly whenever we have parsed results from the various analysis tools and predefined workflows that are executed by the Analysis Server, the Update Server uploads the data into the integrated database.

The volume and complexity of the data, as well as the diversity of the applications being developed at Mathematics and Computer Science division require the data to be stored in a highly integrated fashion. We have developed an integrated relational database which serves as a platform on which we can efficiently develop bioinformatics applications. The integrated database includes sequence and annotation data from public databases NCBI, SwissProt [33], PIR, UniProt [34], and Interpro, as well as metabolic pathway information from 
EMP [35] and KEGG. The database also contains the results obtained from applying different bioinformatics tools to the sequence data, e.g., BLAST, Blocks, and TMHMM. The GADU Update Server ingests and integrates all of this data into an integrated warehouse database, automatically cross-referencing related entities from the various data sources.

\section{Results}

In this section we describe the two significant benefits that have resulted from developing and deploying the GNARE system - namely, the high-throughput analysis of updated genome sequence data that we have achieved with GADU, and the power that GADU and the Integrated Database have provided for building powerful interactive applications.

\subsection{GADU Throughput}

GADU has been used extensively by the computational biology group at Argonne National Laboratory as well as the SEED [36] project, the NIH Midwest Center for Structural Genomics (MCSG) [37] and the NIH Great Lakes RCE [38]. We have developed and continue to develop automated analytical pipelines for the applications in order to manage and submit computationally intensive jobs to the Grid. One such pipeline is a very basic one designed to use the BLAST tool in order to make comparative analysis computations on the complete protein sequence universe. There are constant updates to the protein sequence universe; at this point it is comprised of over 2.1 million amino acid sequences. These sequences are analyzed individually to find out similarities among them which could give clues into how sequences are related with each other. The large number of protein sequences makes these computations very computer-intensive. The following results demonstrate how important using a grid environment for the computations increases the efficiency of the GNARE environment.

Several groups mentioned above have used the BLAST pipeline for their sequence analysis and annotations. One of the first instances of using this BLAST pipeline was for the analysis of the data for the SEED project which at the time consisted of pair-wise comparisons of a database of 1.8 million protein sequences against itself. The complete analysis was performed in 84 hours using 250 nodes from the DOE Science Grid LCRC site. Typically a single BLAST process for one sequence depending on the platform and size of the database may take anywhere from 20 seconds to 60 seconds.
Many more BLAST pipeline invocations have been performed since the first run of 1.8 million sequences and the runs have become more efficient with the addition of more Grid resources (e.g. GRID2003 and TeraGrid). The size of the protein sequence database has also increased to over 2.1 million sequences. The first run mentioned above was not performed with the Site Selector mechanism mentioned above which picked sites from different Grid resource sites. However, the latest runs were performed with the site selector mechanism. The mechanism decreased the pain and suffering of the earlier runs of having to manually select the sites to which to send the analysis. Even though, the quantity of the protein sequence universe has increased, the efficiency of using the site selector has decreased the real time taken to complete the whole database as shown by the results table. This is mainly due to the ability of the site selector testing each site before submitting a job to a particular grid site, therefore, making sure that there are no jobs being submitted to unavailable grid sites. This was one of the main problems in the first set of runs, thus wasting process time. The large differences in run time from the runs using the site selector with the other two type of runs show how much time was wasted re-computing many of the protein sequences that were being submitted to unavailable resources.

\begin{tabular}{|c|c|c|c|}
\hline $\begin{array}{c}\text { Runtime } \\
\text { (hours) }\end{array}$ & $\begin{array}{c}\text { DOE Science } \\
\text { w/o Site Select }\end{array}$ & $\begin{array}{c}\text { GRID3 } \\
\text { w/o Site Select }\end{array}$ & $\begin{array}{c}\text { GRID3, TeraGrid } \\
\text { w/ Site Select }\end{array}$ \\
Pipeline & 1.4 million seq & 1.5 million seq & 1.7 million seq \\
\hline BLAST & 170 & 184 & 108 \\
\hline Blocks & 216 & 224 & 120 \\
\hline
\end{tabular}

The BLAST pipeline is not the only pipeline run using the Grid environment; however, it is the most common one that we run today. Other bioinformatics tools are also run for the complete protein sequence database using different tools (e.g. Blocks, TMHMM) to extract different vector information about the individual protein sequences. These tools are as computer-intensive as the BLAST pipeline and take a similar amount of time to analyze.

\subsection{GNARE Applications}

The use of GNARE has been essential in the development of the following bioinformatics applications:

PUMA2 [39], a system for high-throughput analysis and metabolic reconstruction of genomes from the sequence data. PUMA2 provides a platform for interactive genome functional annotation, metabolic reconstruction and the study of evolution of metabolism and biological function. It contains analyses of over 1000 
completed and partially sequenced organisms through pre-computed sequence analysis results using GNARE.

Pathos [40] provides the bioinformatics support to members of the NIH/NIAID Great Lakes Regional Center of Excellence in Biodefense research.

TarGet [41], a computational environment supporting the NIH Midwest Center for Structural Genomics (MCSG) serves researchers working on selection of protein targets of biomedical importance for the determination of 3D structure.

Sentra [42] provides an interface to a database of prokaryotic Signal Transduction proteins.

MetaGenome serves researchers of the DOE Microbial Genome program [43]. It provides bioinformatics support for the identification and characterization of organisms present in environmental samples taken from the Hanford site.

Chisel [44], provides function prediction, evolutionary and high-resolution analyses of genetic sequence data for enzymatic functions.

In order to provide up-to-date data and analyses to the researchers involved in these projects, given the growth of genomic sequence data, the use of state-of-the-art computational technologies like distributed computing is essential.

\section{Summary and Future Work}

The use of GNARE was essential for the development of the applications in evolutionary analysis of genomes (PUMA2, the SEED), biodefence research (PathosDB), structural biology (TarGet DB) and bioremediation (MetaGenome) that are dependent on availability of upto-date annotations and rely on comparative analysis of large sets of phylogenetically diverse organisms. Use of GNARE allowed for dramatic reduction of time and human resources required for genome analysis. Such increase in efficiency and speed of genome analysis enabled the expert biologists involved in application projects to concentrate on essential biological problems without wasting time and effort on dataprocessing.

Efficiency of GNARE's modular architecture is especially useful for annotation and analysis of newly sequenced genomes. Availability of new experimental results concerning functions of proteins, previously annotated as hypothetical, as well as improvements in the sensitivity and accuracy of bioinformatics tools, requires periodic revisiting of previously annotated genomes and reassignment of functions using this newly acquired knowledge. The increased efficiency of genome analysis offered by the GNARE system and the Grid considerably simplifies the analysis of newly sequenced genomes and re-annotation of previously annotated genomes. GNARE can be an interface to leverage Grid resources for all biologists interested in performing such complex computations. It can hide the complex technologies involved in using distributed Grid resources and help users perform faster and better analyses.

In future work, we plan to advance further the use of Grid technology for the needs of bioinformatics applications in two main areas. First, we plan to provide services to bioinformatics community via a web-based gateway, thus allowing users to submit and analyze their data by variety of tools and algorithms using GNARE as an entry port to the grid environment. It will also enable the users to create customized controlled workflows using GNARE's interface. The development of such server will allow access to advantages offered by the Grid to wide community of researchers that will not be able to use the grid otherwise due to the lack of necessary expertise and resources. A prototype of GNARE web server was already developed. Second, we plan to implement a virtual data warehouse that will use the Grid environment for navigation and analysis of data residing in remote locations.

\section{Acknowledgments}

We extend special thanks to the following individuals who contributed valuable advice and support: Elizabeth Glass, Jens Voeckler, Miron Livny, Zachary Miller, Alain Roy, Susan Coghlan, and the systems support groups of MCS, GRID2003, Globus, Condor, and iVDGL VDT. This work was supported in part by the U.S. Department of Energy under Contract W-31-109-ENG-38, and by the National Science Foundation under grants 86044 (GriPhyN), 122557 (iVDGL), and the NCSA Alliance Expedition “A PACI Petascale Data Quest” (PDQ).

\section{References}

1. GOLD: http://wit.integratedgenomics.com/GOLD/

2. Ideker, T., Galitski, T., Hood, L. (2001) A new approach to decoding life: systems biology. Annu. Rev. Genomics Hum. Genet., 2, 343-372.

3. Altschul, S.F., Madden, T.L., Schaffer, A.A., Zhang, J., Zhang, Z., Miller, W., Lipman, D.J. (1997) Gapped BLAST and PSI-BLAST: a new generation of protein database search programs. Nucleic Acids Res., 25, 33893402.

4. Pearson, W.R. (1994) Using the FASTA program to search protein and DNA sequence databases. Methods Mol Biol., 24, 307-331.

5. Shpaer, E.G., Robinson, M., Yee, D., Candlin, J.D., Mines, R., Hunkapiller, T. (1996) Sensitivity and selectivity in protein similarity searches: a comparison of SmithWaterman in hardware to BLAST and FASTA. Genomics, 38, 179-191. 
6. Mulder, N.J., Apweiler, R., Attwood, T.K., Bairoch, A., Barrell, D., Bateman, A., Binns, D., Biswas, M., Bradley, P., Bork, P., et al. (2003) The InterPro Database, 2003 brings increased coverage and new features. Nucleic Acids Res., 31, 315-318.

7. Bateman, A., Birney, E., Cerruti, L., Durbin, R., Etwiller, L., Eddy, S.R., Griffiths Jones, S., Howe, K.L., Marshall, M., Sonnhammer, E.L. (2002) The Pfam protein families database. Nucleic Acids Res., 30, 276-280.

8. Henikoff, S., Henikoff, J.G., Pietrokovski, S. (1999) Blocks+: a non-redundant database of protein alignment blocks derived from multiple compilations. Bioinformatics, 15, 471-479.

9. Pearl, F.M., Bennett, C.F., Bray, J.E., Harrison, A.P., Martin, N., Shepherd, A., Sillitoe, I., Thornton, J., Orengo, C.A. (2003) The CATH database: an extended protein family resource for structural and functional genomics. Nucleic Acids Res., 31, 452-455.

10. Lo Conte, L., Brenner, S.E., Hubbard, T.J., Chothia, C., Murzin, A.G. (2002) SCOP database in 2002: refinements accommodate structural genomics. Nucleic Acids Res., 30, 264-267.

11. "Encyclopedia of Life” (http://eol.sdsc.edu/)

12. Goble, C., Pettifer, S. and Stevens, R. Knowledge Integration: In silico Experiments in Bioinformatics. The Grid: Blueprint for a New Computing Infrastructure, Morgan Kaufmann, 2004.

13. North Carolina BioGRID (http://www.ncbiogrid.org/)

14. EUROGRID, (http://www.eurogrid.org/)

15. Asia Pacific BioGrid Initiative (http://www.apbionet.org/apbiogrid/).

16. Foster, I. and Kesselman, C. Globus: A Metacomputing Infrastructure Toolkit. International Journal of Supercomputer Applications, 11 (2). 115-128. 1997.

17. Litzkow, M.J., Livny, M. and Mutka, M.W. Condor - A Hunter of Idle Workstations. 8th International Conference on Distributed Computing Systems, 1988, 104-111.

18. Foster, I., Voeckler, J., Wilde, M. and Zhao, Y., The Virtual Data Grid: A New Model and Architecture for Data-Intensive Collaboration. Conference on Innovative Data Systems Research, 2003.

19. Foster, I. and others, The Grid2003 Production Grid: Principles and Practice. IEEE International Symposium on High Performance Distributed Computing, 2004, IEEE Computer Science Press.

20. Catlett, C. The TeraGrid: A Primer, 2002. www.teragrid.org.

21. DOE Science Grid, www.doesciencegrid.org

22. The NCBI handbook [Internet]. Bethesda (MD): National Library of Medicine (US), National Center for Biotechnology Information; 2002 Oct. Chapter 17, The Reference Sequence (RefSeq) Project. Available from http://ncbi.nlm.nih.gov/entrez/query.fcgi?db=Books.

23. Wu, C.H., Huang, H., Arminski, L., Castro-Alvear, J., Chen, Y., Hu, Z., Robert, S., “The Protein Information Resource: an integrated public resource of functional annotation of proteins” 2002, 2002 Oxford University Press Nucleic Acids Research, Vol. 30, No. 1 35-37.

24. Ogata, H., Goto, S., Sato, K., Fujibuchi, W., Bono, H., Kanehis, M., "KEGG: Kyoto Encyclopedia of Genes and Genomes,” 1999, Nucleic Acids Research, Vol 27, No. 1.
25. Altschul, Stephen F., Gish, W., Miller, W., Myers, E.W., Lipman, D.J., "Basic local alignment search tool,” 1990, J. Mol. Biol. 215:403-10.

26. Henikoff, J.G., Henikoff, S., "Blocks Database and its Applications,” 1996, Meth. Enzymology 26:88105. Review article about the BLOCKS database.

27. Bateman, A., Birney, E., Durbin, R., Eddy, S. R., Finn, R. D., Sonnhammer, E. L., "The Pfam protein families database,” 2000, Nucl. Acids Res. 28, 260262.

28. Krogh, Anders, Prediction of transmembrane helices in proteins, http://www.cbs.dtu.dk/services/TMHMM/

29. Foster, J. Voeckler, M. Wilde, and Y. Zhou. Chimera: A virtual data system for representing, querying, and automating data derivation. In Proceedings of the 14th Conference on Scientific and Statistical Database

30. Allcock, W., et al., Data Management and Transfer in High-Performance Computational Grid Environments. Parallel Computing, 2002. 28(5): p. 749-771

31. Ann L. Chervenak, Naveen Palavalli, Shishir Bharathi, Carl Kesselman, Robert Schwartzkopf, Performance and Scalability of a Replica Location Service, Proceedings of the International Symposium on High Performance Distributed Computing Conference (HPDC-13), June 2004.

32. PDB, The Protein Data Bank, http://www.rcsb.org/pdb/

33. Swiss-Prot, The Swiss-Prot Protein Knowledgebase, http://us.expasy.org/sprot/

34. Bairoch A, Apweiler R, Wu C. H., Barker W. C., Boeckmann B., Ferro S, Gasteiger E, Huang H, Lopez R, Magrane M, Martin MJ, Natale DA, O'Donovan C, Redaschi N, Yeh L. S. The Universal Protein Resource (UniProt).

35. Burgard, A.P., Maranas, C.D., "Review of the enzymes and metabolic pathways (emp) database," 2001, Metab. Eng. 3: 193-194.

36. Ross Overbeek, Terry Disz, Rick Stevens, The SEED: a peer-to-peer environment for genome annotation, Communications of the ACM Volume 47, Number 11 (2004), pages 46-51.

37. Midwest Center for Structural Genomics (MCSG) http://www.mcsg.anl.gov

38. Great Lakes Regional Center of Excellence for Biodefense \& emerging Infectious Diseases Research, http://www.glrce.org.

39. PUMA2 System, http://compbio.mcs.anl.gov/puma2

40. Pathos System, http://compbio.mcs.anl.gov/pathos

41. TarGet Environment, http://compbio.mcs.anl.gov/target

42. Natalia Maltsev, Elizabeth Marland, Gong-Xin Yu, Saurabha Bhatnagar, and Richard Lusk. Sentra, a database of signal transduction proteins. Nucleic Acids Res, 2002, Vol. 30. http://www-wit.mcs.anl.gov/sentra

43. DOE Microbial Genome Program, http://microbialgenome.org

44. Chisel, http://compbio.mcs.anl.gov/CHISEL 